\title{
INTEGRATING AIR FLOW COUNTER FOR HIGH VOLUME AIR SAMPLER
}

\author{
Kosuke NOZAKI \\ National Institute of Industrial Health, Kizuki-Sumiyoshi, Kawasaki
}

(Received March 30, 1965)

\begin{abstract}
The integral air flow counter was designed for application to the high volume air sampler.

The static pressure of the air at the outlet of high volume air sampler was picked up by the transducer.

The strain of thin plate of phosphorbronze stretched in the cylindrical vessel was transformed into electrical potential by the differential transformer.

The continuous value of the electrical potential was transformed to the digital value by the integrated circuit, and this digital pulse was counted for measuring the air volume.

Additionally, the instantaneous value of the air flow can be indicated by the meter.
\end{abstract}

The high volume air sampler with filter paper sheets or glass filter sheets is widely used for the collection of air borne particles.

To estimate the dust concentration in the air from the dust collected on the filter sheets, needs the air volume passing through the filter.

At present, the flow meter used for the high volume air sampler indicates the only instantaneous air flow rate1).

As the high volume air sampler is operated usually for a long period to collect the particulate matter in the atmosphere, the flow rate decreases gradually with time by clogging of the filter.

The change of the flow rate may be classified into the following three factors.

1. Difference of concentration of particulate matter in the atmospheric or industrial environment2).

Flow rate is changed by the concentration of particles in the air, because the amounts of deposited particles with which the filter is clogged depend on the particle concentration.

2. Difference of clogging effect of filters.

Even if the dust concentration does not change, the clogging rate is changed by the difference of filtering mechanism of the filter.

3. Difference of characteristics of suction pump. 


\section{INTEGRATING AIR FLOW COUNTER}

If the static pressure of suction pump is high enough, the decrease of the flow rate by the clogging effect does not appear, but the suction pump applied to high volume air sampler has no such high static pressure.

Therefore, the total air volume can not be measured accurately without continuous observation of the flow meter.

In order to overcome these difficulties, the apparatus which can measure both the instantaneous value of the air flow and total air volume passing through the filter of the high volume air sampler, was designed.

\section{Procedures}

The $\mathrm{U}$ tube manometer is connected at the outlet of the high volume air sampler. Then, the change of air flow passing through the sampler is transformed to change of pressure in the analogue value.

Using the circuit showed in Fig. 1, the change of pressure is transformed to the electric current. This electric current is integrated and when the potential of condencer C reaches to a fixed value one electric pulse is produced. By this method, integrated air volume can be easily measured by counting of pulses produced for a given time.

In the flow meter of differential type such as a $U$ tube manometer the relation between the passed air volume and the pressure difference is shown by the following equation.

$$
\mathrm{Q}=\mathrm{c} \sqrt{\mathrm{H}}
$$

where, $\mathrm{Q}=$ air volume, $\mathrm{H}=$ pressure difference, $\mathrm{c}=$ constant.

So, the value of $Q$ and $H$ is not linear.

Methods for the change of the non-linearity between the quantity $Q$ and $H$ are as follows.

1. Use of non-linear element.

2. Use of cam or spring which has the square root output characteristics for input3).

3. Use of the feed-back electric circuit by which square root of the output voltage is corresponded to the input voltage.

And then, the combined transducer with the thin plate of phosphorbronze and the differential transformer was used.

This transducer was used in such a region as there exists a proportional relation between the strain of phosphorbronze thin plate and the pressure difference of the orifice.

And this condition was easily accomplished by the adjustment of the orifice connected to high volume air sampler.

The electric circuit for the measurement of air flow is shown in Fig. 1.

The grade of strain was picked up by the differential transformer as an electric potential. The high freqency voltage produced by the oscillator $o$ in Fig. 1 was supplied to the primary coil of the differencial transformer $\mathrm{T}$ as a carrier wave. The amplitude of this carrier wave was modurated by the strain. The amplitude modurated carrier wave which was proportional to the strain of the thin plate was produced in the secondary coil of the differencial transformer. This signal was rectified, and charged to the condenser $\mathrm{C}$ as shown in Fig. 1. 

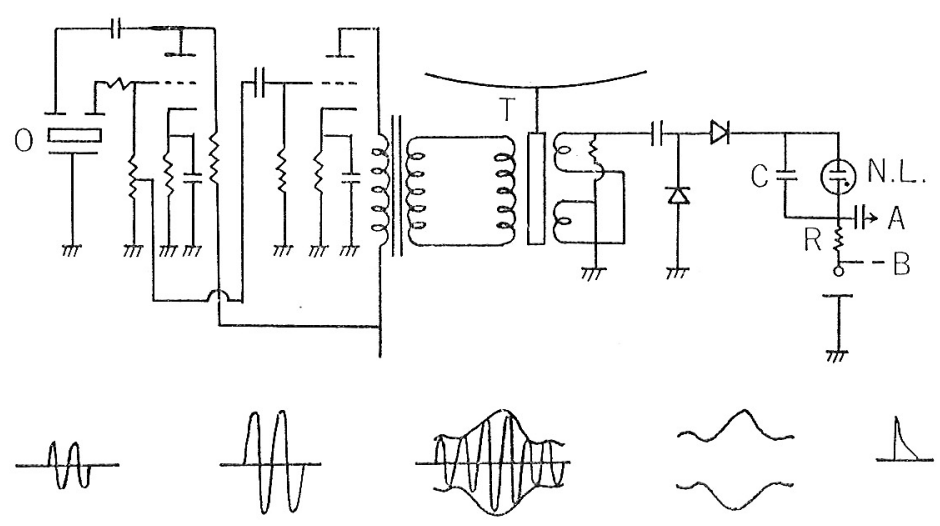

Fig. 1. The electric circuit of the translucer.

The condenser $\mathrm{C}$ was discharged through the neon tube (N. L.) when the electric potential reached to a certain value. At this moment, potential difference was produced at both end of the resistor $R$. Then the pulse was obtained at the point $A$ in Fig. 1. This pulse had a
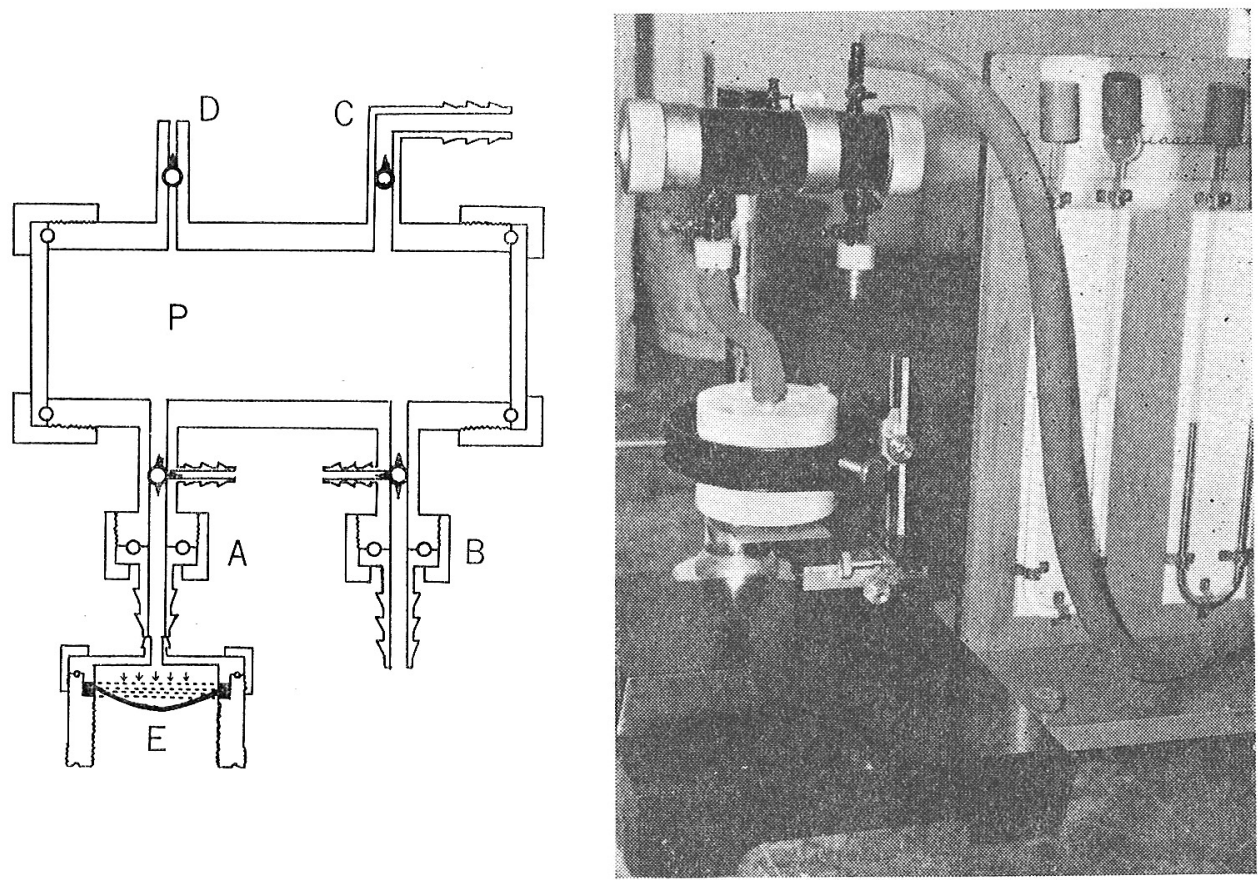

Fig. 2. Photograph and section of pressure container.

height equal to the difference between the discharge voltage of the neon tube and the negative voltage of the Fig. 1 and preservation potential of neon tube, and a width dependent 


\section{INTEGRATING AIR FLOW COUNTER}

on the capacity of condenser $\mathrm{C}$ and the resistor $\mathrm{R}$. This pulse was integrated by the electrical circuit in order to determine the total air volume.

\section{Characteristics of Transducer}

The H. E. type (high elasticity) phosphorbronze thin plate of about $0.15 \mathrm{~mm}$ thickness was strained by loading pressure to one side of the plate.

To examine the characteristics of strain of the H. E. type phosphorbronze following devices were used. Uniformly distributed pressure was loaded to the thin plate by the pressure container and the strain of the thin plate was measured by travelling microscope. The pressure loaded to the thin plate was determined by the manometer. The pressure container is shown in Fig. 2.

The pressure container $\mathrm{P}$ has an inlet $\mathrm{C}$, leak cock $\mathrm{D}$ and two outlets $\mathrm{A}$ and $\mathrm{B}$. Outlet A was connected to the cylindrical vessel $\mathrm{E}$ in which the phosphorbronze thin plate was stretched. Outlet B was connected to the manometer for the measurement of pressure in the cylindrical vessel.

The inlet $\mathrm{C}$ was connected to the compressor. The pressure in the cylindrical vessel was adjusted by the leak cock D. The cross section of the cylindrical vessel $\mathrm{E}$ is shown in Fig. 3. The distance between the differential transformer $\mathrm{T}$ and thin plate $\mathrm{P}$ was adjusted by ring $\mathrm{R}$ and the center of core of the differential transformer was adjusted by three screws $\mathrm{S}$. The strain of thin plate was measured by the travelling microscope.

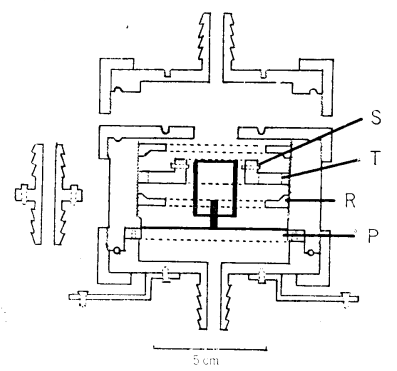

Fig. 3. Section of the cylindrical vessel E.

$T$ : Fix ring of differential transformer.

$\mathrm{R}:$ Distance adjust ring for differential transformer.

$\mathrm{S}:$ Screw for center adjust.

$\mathrm{P}:$ Fix ring for thin plate.

The relation between the strain and the pressure is shown in Fig. 4. From this experimental result, it is clear that the strain of phosphorbronze thin plate is proportional to square root of the pressure when the grade of strain is under $1.3 \mathrm{~mm}$.

The strain was transmitted to the core of the differential transformer. This transformer was used in the region of strain below $1.3 \mathrm{~mm}$.

The output of differential transformer of type 36-SM was linearly proportional to the displacement of the core in the range of the displacement of $\pm 4 \mathrm{~mm}$ from the neutral 
K. NOZAKI

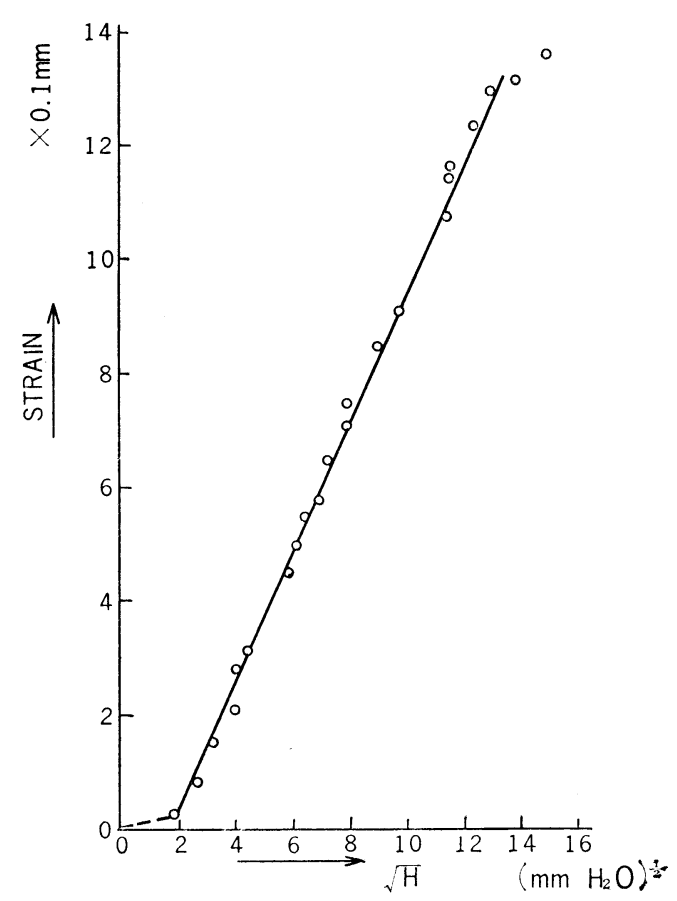

PRESSURE DIFFERENCE

Fig. 4. Relation between the pressure and the strain of the thin plate.

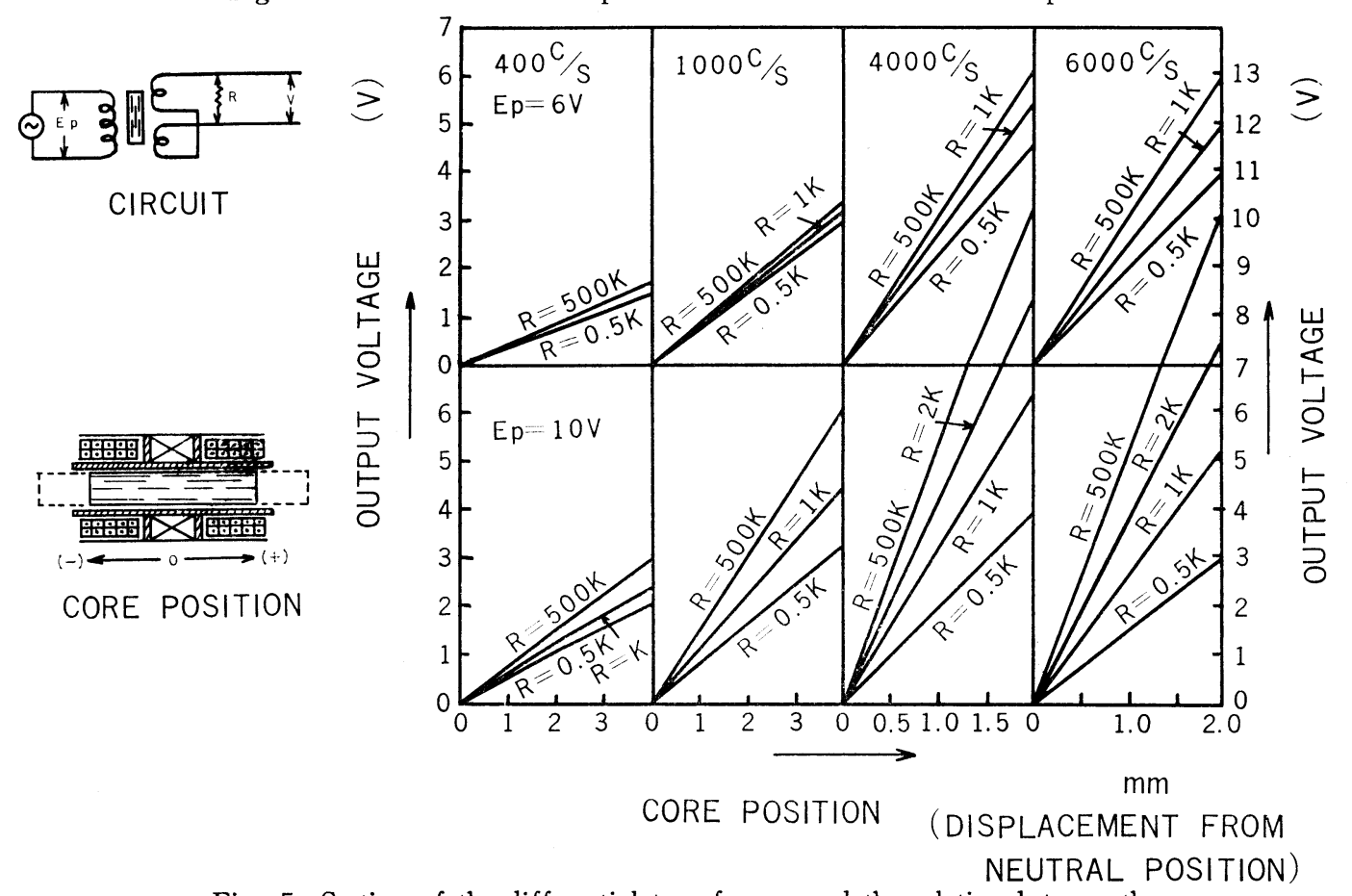

Fig. 5. Section of the differential transformer and the relation between the output voltage and core position. 


\section{INTEGRATING AIR FLOW COUNTER}

position. In order to obtain the optimum voltage and frequency of carrier wave supplied to the primary coil of the differential transformer output voltage of differential transformer was measured under the several conditions of voltage and frequency of carrier wave and core positions. Experimental results are shown in Fig. 5. In this figure, Ep is voltage of carrier wave and $\mathrm{R}$ is loading resistance of output circuit of the differential transformer. Besides, the input optimum voltage and frequency were obtained considering the relation between the strain of thin plate and the output voltage of differential transformer. The carrier wave of 4000 $\mathrm{c} / \mathrm{s} \quad \mathrm{Ep}=6 \mathrm{~V}$ and the loading resistance of over $500 \mathrm{k} \Omega$ was found to be optimum. The pulse number of the output signal of the differential transformer which correspond to the air volume was counted by the circuit shown in Fig. 6. The number of pulses was read out by two Decatrons and mechanical register.

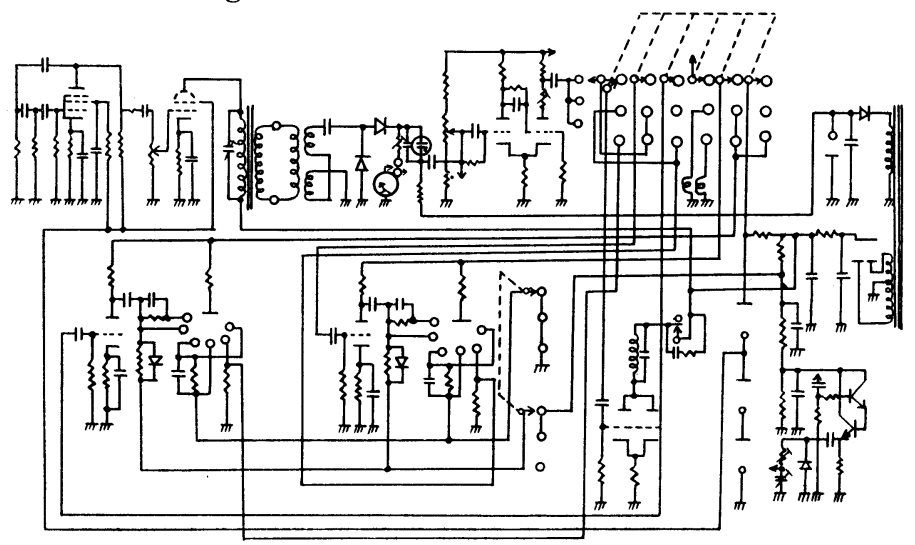

Fig. 6. The electric circuit of the integrating air flow counter.

The procedure of calibration between the number of pulses and the sampling air volume is as follows. As high volume air sampler modified vacuum cleaner for industrial use was used. The wet gasmeter and poly-ethylene balloons of capacities of $1,3,5,15$, and $30 \mathrm{~m}^{3}$ were used

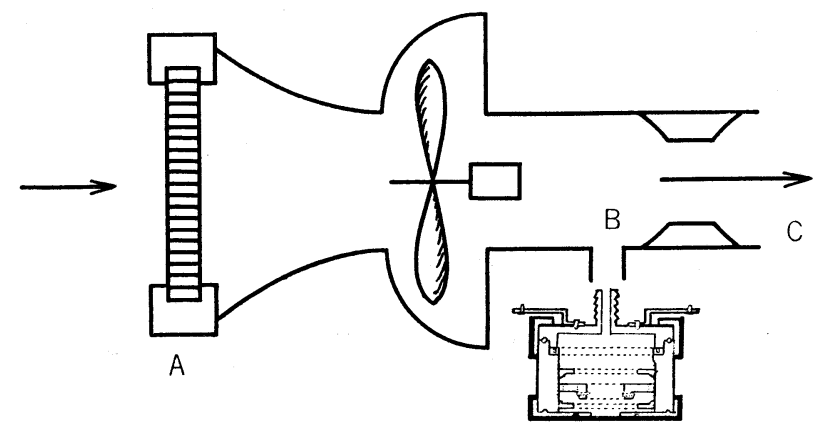

Fig. 7. The flow sheet of the method of calibration.

A : filter holder.

$\mathrm{B}:$ to the detector.

C : air outlet.

for the calibration of this device. The flow sheet of the method of calibration is shown in Fig. 7. The sampling air was suctioned by the fan passing through the filter and the detector 


\section{K. NOZAKI}

which was connected to the side of outlet tube as in Fig. 7. To load the various flow resistances to the inlet of this device, the plates with various total areas of evenly distributed holes were mounted to the filter holder instead of filter.

And the air passing through the resistance plate instead of air filter was kept in the polyethylene balloon. The air volume in the poly-ethylene balloon was measured by wet gas meter.

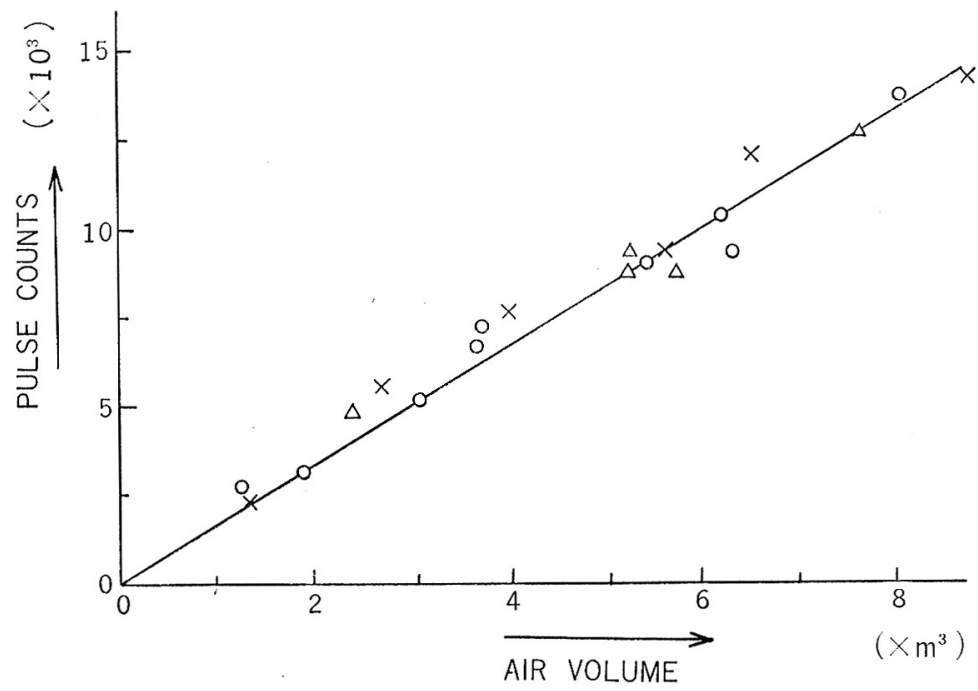

Fig. 8. The relation between the air volume and number of counts of the integrating air flow counter.

symbols $\bigcirc$ correspond, to the filter of high resistance,

$\triangle$ to the filter of moderate resistance,

$x$ to the filter of low resistance.

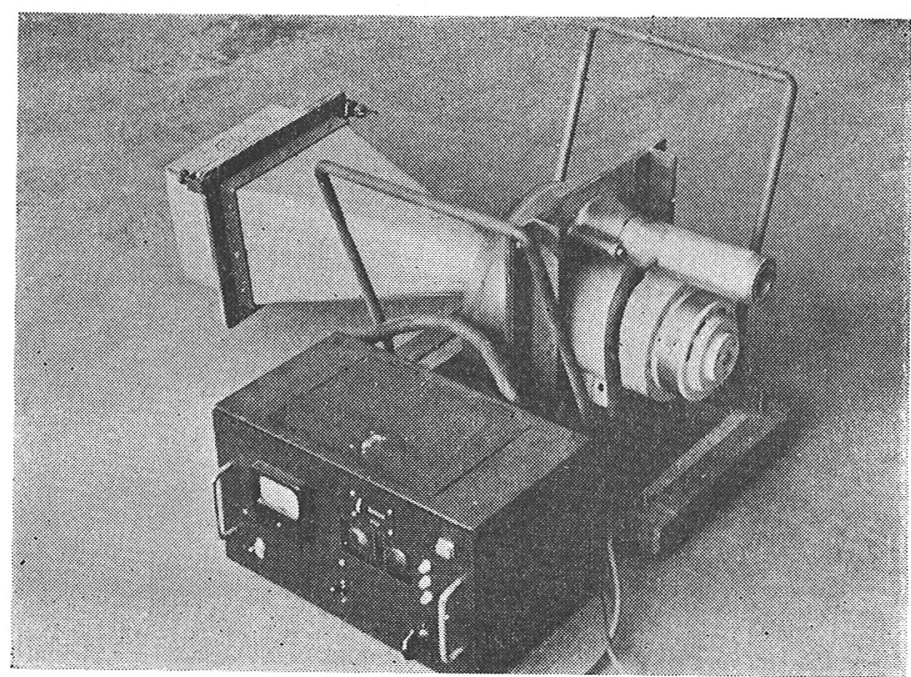

Fig. 9. Photograph of the apparatus. 


\section{INTEGRATING AIR FLOW COUNTER}

The counted number of pulse against the sampling air volume is shown in Fig. 8. This apparatus showed 1700 counts against $1 \mathrm{~m}^{3}$ of sampling air volume passing through the filter. Fig. 9 is the photograph of the device.

\section{Application}

The apparatus was applied to the long-time sampling of atmospheric particulate matter. As the filter media, the glass fiber filter with high efficiency was used. The initial flow rate was $1.4 \mathrm{~m}^{3} / \mathrm{min}$ and the flow rate decreased to $0.6 \mathrm{~m}^{3} / \mathrm{min}$ after 48 hours running, and the total sampling air volume was $2500 \mathrm{~m}^{3}$.

In this running, the atmospheric particulate matter of $500 \mathrm{mg}$ was collected on the filter surface.

\section{REFERENCES}

1) Yaffe, C. D., Byers, D. H. and Hosey, A. D. (1956). Encyclopedia of Instrumentation for Industrial Hygiene, pp. 90, 92, 619, 621, University of Michigan, Ann Arbor.

2) Harrison, W. K. Jr., Nader, J. S. and Fugman, F. S. (1960). Am. Ind. Hyg. Assoc. J., 21, 115 .

3) Hisada, K. (1962). J. Soc. Instr. Control Engr., 1, 135. (in Japanese). 